\title{
Matrix Pencil for Direction Of Arrival Using Smart Antenna
}

\author{
Mohammed Amine Ihedrane ${ }^{\# 1}$, SeddikBri $^{\# 1}$ \\ ${ }^{\#}$ Materials and instrumentation (MIM), Electrical Engineering Department, \\ High School of Technology, MoulayIsmail University, Meknes-Morocco. \\ amine.ihedrane@gmail.com, briseddik@gmail.com
}

\begin{abstract}
The main purpose of the smart antenna system is the selection of smart algorithms for adaptive array to estimate the Direction Of Arrival (DOA). By using Matrix Pencil (MP) algorithms the weight of the antenna array can be adjusted to form certain amount of adaptive beam to track corresponding users automatically and to minimize interference arising from others by introducing nulls in their direction. The interference can be suppressed and the desire signal can be extracted. One of the adaptive Matrix Pencil algorithm Least Mean Square Algorithm (LMS) is presented on this article using Uniform Circular Array (UCA) structure. Smart antenna incorporates this algorithm in coded form which calculates complex weights according to the signal environment. The efficiency of (LMS) algorithm is compared on the basis of normalized array factor and Root Mean Square Error (RMSE) for mobile communication. The results demonstrate clearly that the Matrix Pencil investigate in this work is more accurate and stable compared to the published measure.
\end{abstract}

Keyword-Smart Antenna, DOA, LMS, MP, RMSE, UCA

\section{INTRODUCTION}

Smart antenna has been widely used in many applications such as radar, sonar and wireless communication systems. Considerable research efforts have been made to estimate the direction of arrival (DOA) and various array signal process techniques for DOA estimation have been proposed. In particular, the DOA estimation for uniform circular arrays (UCAs) has been developed in these scenarios, which desired all-azimuth angle coverage. By the virtue of their geometry, UCAs are able to provide $360^{\circ}$ of coverage in azimuth plane. Moreover, they are known to be is isotropic. That is, they can estimate the DOA of incident signal with uniform resolution in the azimuth plane. In addition, direction patterns synthesized with UCAs can be electronically rotated in the plane of the array without significant change of beam shape [1]-[4].

There are various methods to estimate the angle of arrival (DOA) of radio signals on the antenna array. DOA estimation techniques can be broadly divided into three different categories namely; conventional methods subspace based methods and maximum likelihood methods. Convolution methods are based on the concepts of beam forming and null steering, but it requires a large number of elements to provide high resolution. Examples of this method are delay and sum and Capon's minimum variance method [2].

One major limitation of this method is poor resolution that is its ability to separate closely spaced signals. Unlike conventional methods, subspace methods exploit the information of the received data resulting in high resolution. Two main subspace based algorithms are Multiple Signal Classification and Estimation of Signal Parameters via Rotational Invariance Techniques. The DOA algorithms are classified as quadratic (non subspace) type and subspace type. The Barltett and Capon (Minimum Variance Distortion less Response) [3] are quadratic type algorithms. Both methods are highly dependent on physical size of array aperture, which results in poor resolution and accuracy. Subspace based DOA estimation method is based on the eigen decomposition. The subspace based DOA estimation algorithms MUSIC and ESPRIT provide high resolution; they are more accurate and not limited to physical size of array aperture.

Others techniques involve modification of the covariance matrix through a pre-processing scheme called spatial smoothing [5]. Sarkar and Hua [6][7] utilized the MP to get the DOA of the signals in a coherent multipath environment. In the MP method, based on the spatial samples of the data, the analysis is done on a snapshot-by-snapshot basis [8-9], and hence is computationally quite efficient. A snapshot is defined as the voltages measured at the feed points of all the antenna elements in the array at a particular instance of time. Non-stationary in the data then has little effect for this method, as no assumption is made about the statistics of the environment. Unlike the conventional covariance matrix techniques, the MP method can find DOA easily in the presence of multipath coherent signal without performing additional processing of spatial smoothing. This methodology is based on the assumption that each antenna element is isotropic point radiator and is spaced uniformly along a line for 1D-DOA and on plane for 2D-DOA

In this article, we present a DOA estimation procedure for $\mathrm{M}$ correlated signals impinging on a uniform circular array of $\mathrm{N}$ elements using Matrix Pencil. The stochastic gradient algorithm LMS (Least Mean Squares) was used to guide the radiation pattern of the antenna to the desired signal. For the circular array, we have 
analysed the performances of theproposed algorithms (number of antenna elements, number of snapshots and spacing between elements) and compared them with the published measure.

\section{MATRIX PENCIL BASED ON UNIFORM LINEAR ARRAY}

The Matrix Pencil formulations use the real Matrices to estimate the DOA of multiple signals simultaneously impinging on the ULA [10]. The vector $\mathrm{x}(\mathrm{n})$ is the set of voltages measured at the feed point of antenna element of the ULA. Therefore, $x(t)$ can be modelled by a sum of complex exponentials [11]. The observed voltage is given by:

$$
\mathrm{y}(\mathrm{t})=x(t)+n(t)=\sum_{i=1}^{N} \mathrm{R}_{\mathrm{i}} e^{\mathrm{s}_{\mathrm{i}} t}+\mathrm{n}(\mathrm{t})(1)
$$

$y(t)=$ observed voltages at a specific instance $t$

$\mathrm{n}(\mathrm{t})=$ noise associated with the observation

$\mathrm{x}(\mathrm{t})=$ actual noise free signal

Therefore, one can write the sampled signal as,

$$
y(P)=\sum_{i=1}^{N} R_{i} z_{i}^{p}+n(p), \text { for } p=0,1, \ldots, N-1
$$

Where,

$$
Z i=e^{j \frac{2 \pi}{\lambda} d \sin (\theta)}, \text { for } i=1,2, \ldots, N
$$

In this presentation, it has been assumed that the damping factor $\alpha_{i}$ is equal to zero. The objective is to find the best estimation for $\theta$. Let us consider the matrix $Y$ which is obtained directly from $\mathrm{x}(\mathrm{p})$. Y is a Hankel matrix, and each column of $Y$ is a windowed part of original data vector, $\{\mathrm{x}(0) \times(1) \times(2) \ldots \times(\mathrm{N}-1)\}$.

$$
\mathrm{Y}=\left[\begin{array}{cccc}
\mathrm{x}(0) & \mathrm{x}(1) & \ldots & \mathrm{x}(\mathrm{L}-1) \\
\mathrm{x}(1) & \mathrm{x}(2) & \ldots & \mathrm{x}(\mathrm{L}) \\
\vdots & \vdots & \ddots & \vdots \\
\mathrm{x}(\mathrm{N}-\mathrm{L}) & \mathrm{x}(\mathrm{N}-\mathrm{L}+1) & \ldots & \mathrm{x}(\mathrm{N}-\mathrm{L})
\end{array}\right](4) N-(L+1) x(L)
$$

The parameter $L$ is called the pencil parameter. $L$ is chosen between $\mathrm{N} / 3$ and N/2 for efficient noise filtering [12]- [13]. The variance of the estimated values of $\mathrm{R}_{\mathrm{i}}$ and $\mathrm{Z}_{\mathrm{i}}$ will be minimal if the values of $L$ are chosen in this range [14]. From the matrix $Y$, we can define two sub-matrixes, say

$$
\begin{aligned}
& \mathrm{Y}_{\mathrm{a}}=\left[\begin{array}{cccc}
\mathrm{x}(0) & \mathrm{x}(1) & \ldots & \mathrm{x}(\mathrm{L}-1) \\
\mathrm{x}(1) & \mathrm{x}(2) & \ldots & \mathrm{x}(\mathrm{L}) \\
\vdots & \vdots & \ddots & \vdots \\
\mathrm{x}(\mathrm{N}-\mathrm{L}-1) & \mathrm{x}(\mathrm{N}-\mathrm{L}) & \ldots & \mathrm{x}(\mathrm{N}-2)
\end{array}\right](5) \\
& (N-L) x(L) \\
& \mathrm{Y}_{\mathrm{b}}=\left[\begin{array}{cccc}
\mathrm{x}(1) & \mathrm{x}(1) & \ldots & \mathrm{x}(\mathrm{L}-1) \\
\mathrm{x}(2) & \mathrm{x}(2) & \ldots & \mathrm{x}(\mathrm{L}) \\
\vdots & \vdots & \ddots & \vdots \\
\mathrm{x}(\mathrm{N}-\mathrm{L}) & \mathrm{x}(\mathrm{N}-\mathrm{L}+1) & \ldots & \mathrm{x}(\mathrm{N}-1)
\end{array}\right](6) \\
& (N-L) x(L)
\end{aligned}
$$

We can also write

$$
\begin{gathered}
\mathrm{Y}_{\mathrm{a}}=\mathrm{Z}_{\mathrm{a}} \mathrm{R} \mathrm{Z}_{\mathrm{b}}(7) \\
\mathrm{Z}_{\mathrm{a}}=\left[\begin{array}{cccc}
1 & \mathrm{Y}_{\mathrm{b}}=\mathrm{Z}_{\mathrm{a}} \mathrm{R}_{0} \mathrm{Z}_{0} \mathrm{Z}_{\mathrm{b}}(8) \\
\mathrm{Z}_{1} & \mathrm{Z}_{2} & \ldots & \mathrm{Z}_{\mathrm{M}} \\
\vdots & \vdots & \ddots & \vdots \\
\mathrm{Z}_{1}{ }^{(\mathrm{N}-\mathrm{L}-1)} & \mathrm{Z}_{2}^{(\mathrm{N}-\mathrm{L}-1)} & \ldots & \mathrm{Z}_{\mathrm{M}}^{(\mathrm{N}-\mathrm{L}-1)}
\end{array}\right](9) \\
(N-L) x(L)
\end{gathered}
$$


Now, we consider the Matrix Pencil

$$
\mathrm{Y}_{\mathrm{b}}-\lambda \mathrm{Y}_{\mathrm{a}}=\mathrm{Z}_{\mathrm{a}}-\mathrm{R}_{0}\left[\mathrm{Z}_{0}-\lambda \mathrm{I}\right] \mathrm{Z}_{\mathrm{b}}(13)
$$

Here $I$ is the $(\mathrm{MxM})$ identity matrix, one can show that the rank of $\mathrm{Yb}-\lambda$ Ya will be $\mathrm{M}$, provided that $\mathrm{M} \leq \mathrm{L} \leq$ N-M [15-16]. However, if $\lambda=\mathrm{Zi}, \mathrm{i}=1,2, \ldots, \mathrm{M}$ the ith row of $\left[\mathrm{Z}_{0}-\lambda \mathrm{I}\right]$ is zero, then the rank of this matrix is $(\mathrm{Mx} 1)$. Therefore, the parameters $\mathrm{Zi}$ can be found as the generalized eigenvalues of the matrix pair $\left\{\mathrm{Ya}^{+} \mathrm{Yb}-\lambda \mathrm{I}\right\}$ where $\mathrm{Ya}^{+}$is the Moore-Penrose pseudo inverse of Ya, which is defined as :

$$
\mathrm{Y}_{\mathrm{a}}{ }^{+}=\left\{\mathrm{Y}_{\mathrm{a}}{ }^{\mathrm{H}} \mathrm{Y}_{\mathrm{a}}\right\}^{-1} \mathrm{Y}_{\mathrm{a}}{ }^{\mathrm{H}}(14)
$$

The DOA is obtained from

$$
\theta_{\mathrm{i}}=\sin ^{-1}\left(\frac{\operatorname{Im}\left(\log Z_{\mathrm{i}}\right)}{\pi \mathrm{d}}\right)(15)
$$

Where $\mathrm{Zi}$ is defined in eq. (3)

\section{MATRIX PENCIL USING UNIFORM CIRCULAR ARRAY}

We consider a circular array of isotropic antennas evenly spaced $d_{x}$ and $d_{y}$ respectively along the axes $O X$ and $\mathrm{OY}$, where we assume $\mathrm{dx}=\mathrm{dy}=\mathrm{d}$. The network receives signals Ms with the angles of incidence $\left(\theta_{\mathrm{q}}, \varphi_{\mathrm{q}}\right)$, which are respectively $\varphi_{\mathrm{q}}, \theta_{\mathrm{q}}$ and the directions of arrival in elevation and in azimuth. The information on the arrival direction is contained in the eigenvalues of the two transformation matrices that bind respectively subnets 1 and 2 in the $X$ direction and subnets $3 \& 4$ in the Y-direction [17]-[18]. The values of $\alpha_{x}$ and $\alpha_{y}$ are written in the following form:

$$
\begin{aligned}
\alpha_{\mathrm{xi}}=\exp \left(\mathrm{j}\left(\frac{2 \pi \Delta}{\lambda_{0}}\right) \sin \theta \mathrm{i} \cos \Phi \mathrm{i}\right) \\
\alpha_{\mathrm{xi}}=\exp \left(\mathrm{j}\left(\frac{2 \pi \Delta}{\lambda_{0}}\right) \sin \theta \mathrm{i} \sin \Phi \mathrm{i}\right)(17)
\end{aligned}
$$

The elevation and azimuth are expressed by the following equation:

$$
\begin{gathered}
\theta i=\operatorname{Arcsin}\left[\left(\frac{-\mathrm{j} \lambda_{0}}{2 \pi \Delta}\right) \sqrt{\left(\operatorname{Ln} \alpha_{\mathrm{xi}}\right)^{2}+\left(\operatorname{Ln} \alpha_{\mathrm{yi}}\right)^{2}}\right] \\
\Phi \mathrm{i}=\operatorname{Arctg}\left(\frac{\operatorname{Ln} \alpha_{\mathrm{xi}}}{\operatorname{Ln} \alpha_{\mathrm{yi}}}\right)(19)
\end{gathered}
$$

Where $\mathrm{i}=1,2, \ldots$, Ms.

\section{RESULTS AND DISCUSSION}

\begin{tabular}{|c|c|c|c|c|}
\hline \multicolumn{2}{|c|}{ Number of elements } & $\theta_{\text {in }}$ & $\boldsymbol{\theta}_{\text {pencil }}$ & $\Delta \theta_{\text {pencil }}$ \\
\hline \multirow{2}{*}{7} & Pencil [19] & $\begin{array}{l}30 \\
60 \\
\end{array}$ & $\begin{array}{l}37.5900 \\
61.8400\end{array}$ & $\begin{array}{l}0.253 \\
0.030\end{array}$ \\
\hline & This Work & $\begin{array}{l}30 \\
60\end{array}$ & $\begin{array}{l}30.0017 \\
60.0928\end{array}$ & $\begin{array}{l}0.226 \\
0.025\end{array}$ \\
\hline \multirow{2}{*}{8} & Pencil [19] & $\begin{array}{l}30 \\
60 \\
\end{array}$ & $\begin{array}{l}30.1800 \\
61.6400\end{array}$ & $\begin{array}{l}0.006 \\
0.027\end{array}$ \\
\hline & This work & $\begin{array}{l}30 \\
60\end{array}$ & $\begin{array}{l}30.0477 \\
60.0267\end{array}$ & $\begin{array}{l}0.003 \\
0.018\end{array}$ \\
\hline \multirow{2}{*}{10} & Pencil [19] & $\begin{array}{l}30 \\
0.2\end{array}$ & $\begin{array}{c}30.3100 \\
0.2100\end{array}$ & $\begin{array}{c}0.010 \\
0.05\end{array}$ \\
\hline & This Work & $\begin{array}{l}30 \\
0.2\end{array}$ & $\begin{array}{c}30.0004 \\
0.2010\end{array}$ & $\begin{array}{l}0.006 \\
0.005\end{array}$ \\
\hline \multirow{2}{*}{14} & Pencil [19] & $\begin{array}{l}30 \\
60\end{array}$ & $\begin{array}{l}30.1700 \\
59.4900\end{array}$ & $\begin{array}{c}0.005 \\
0.0085\end{array}$ \\
\hline & This Work & $\begin{array}{l}30 \\
60 \\
\end{array}$ & $\begin{array}{l}29.9975 \\
60.0009\end{array}$ & $\begin{array}{r}0.0026 \\
-0.0033 \\
\end{array}$ \\
\hline
\end{tabular}

In this section, the simulations results illustrate the performance of the Matrix Pencil method. The noisy signal model is formulated from (10). $\mathrm{n}(\mathrm{k})$ id treated as a zero mean Gaussian whit noise with variance $\sigma^{2}$.the distance between any two elements of the smart antenna is half wavelength.y(k) is the voltage induced at each of the antenna elements, for $\mathrm{k}=0,1, \ldots, \mathrm{N}-1$. In order to demonstrate the numerical properties, a comparative study was made between the Matrix Pencil and Pencil Method indicates in [19]-[20]-[21]-[22].

TABLE I. COMPARATIVE RESULTS FOR MATRIX PENCIL WITH VARIOUS ELEMENTS 
Table I illustrates the comparison between Matrix Pencil investigate in this research work using UCA and Matrix Pencil indicate at [19]. The comparison show that the authors in [19] indicate that the Matrix Pencil can accuracy estimate the direction of arrival of signal on smart antenna only with one snapshot but the pencil method studied in this work gives more precision than [20] because we have chose a better value of parameter of pencil ' $\mathrm{L}$ ' to increases the precision and the uniform circular arrays have better performance than the other geometry.

TABLE II. COMPARATIVE RESULTS FOR MATRIX PENCIL FOR 100 AND 95 ELEMENTS

\begin{tabular}{|c|c|c|c|c|}
\hline \multicolumn{2}{|c|}{ Number of elements } & $\boldsymbol{\theta}_{\text {in }}$ & $\theta_{\text {out }}$ & $R M S E$ \\
\hline \multirow{12}{*}{100} & \multirow{6}{*}{ Pencil [20] } & 0 & 0.0004 & \multirow{6}{*}{0.0019} \\
\hline & & 5 & 5.0001 & \\
\hline & & 10 & 9.9948 & \\
\hline & & 15 & 15.0014 & \\
\hline & & 20 & 20.0045 & \\
\hline & & 30 & 29.9984 & \\
\hline & \multirow{6}{*}{$\begin{array}{l}\text { This } \\
\text { Work }\end{array}$} & 0 & 0.0000 & \multirow{6}{*}{0.0006} \\
\hline & & 5 & 5.0001 & \\
\hline & & 10 & 9.9991 & \\
\hline & & 15 & 15.0001 & \\
\hline & & 20 & 20.0000 & \\
\hline & & 30 & 30.0001 & \\
\hline \multirow{12}{*}{95} & \multirow{6}{*}{ Pencil [20] } & 0 & 0.0067 & \multirow{6}{*}{0.0023} \\
\hline & & 5 & 5.0024 & \\
\hline & & 10 & 10.0014 & \\
\hline & & 15 & 14.9976 & \\
\hline & & 20 & 19.9965 & \\
\hline & & 30 & 29.9991 & \\
\hline & \multirow{6}{*}{ This work } & 0 & 0.0005 & \multirow{6}{*}{0.0018} \\
\hline & & 5 & 5.0006 & \\
\hline & & 10 & 10.0008 & \\
\hline & & 15 & 15.0009 & \\
\hline & & 20 & 20.0000 & \\
\hline & & 30 & 29.9999 & \\
\hline
\end{tabular}

*RMSE is the Root Mean square Error is defined as follows: RMSE $=\sqrt{\mathrm{E}\left(\theta_{\mathrm{Act}}-\theta_{\mathrm{Mes}}\right)^{2}}$. Where $\mathrm{E}$ is the mean value, $\theta_{\text {Act }}$ is the actual DOA and $\theta_{\text {Mes }}$ is the estimated values.

In Table II, the MP given in this work gives a good precision compared to the proposed one indicate at [20]. The authors in [20] didn't give the number of snapshots using in this case contrary to the second case when they use 100 element with 5 faulty elements they used one snapshots to demonstrate the efficiency of their proposed method, but inour case we have chosen the same number of snapshots in order to improve the robustness of our method with only one snapshots even if the number of elements changed or one of them stops working.

In following step, we compare the LMS investigate at this work and LMS indicate at [21]-[22]. In the Fig. 1 we observe that the final weighted array which has a sharp peak at the desired direction of $0^{\circ}$ and a null at the interfering direction of $-60^{\circ}$. The maximum lobe is observed in the desired direction which is given as an input, also minimum lobe or nulls are observed in noise directions. From Fig. 2 it is observed that the array output acquires and tracks the desired signal after 20 iterations contrary to the results show at [21]-[22]. The results presented in Figs. 2 indicate that if the signal characteristics are rapidly changing, the LMS algorithm may not allow tracking of the desired signal in a satisfactory manner. 


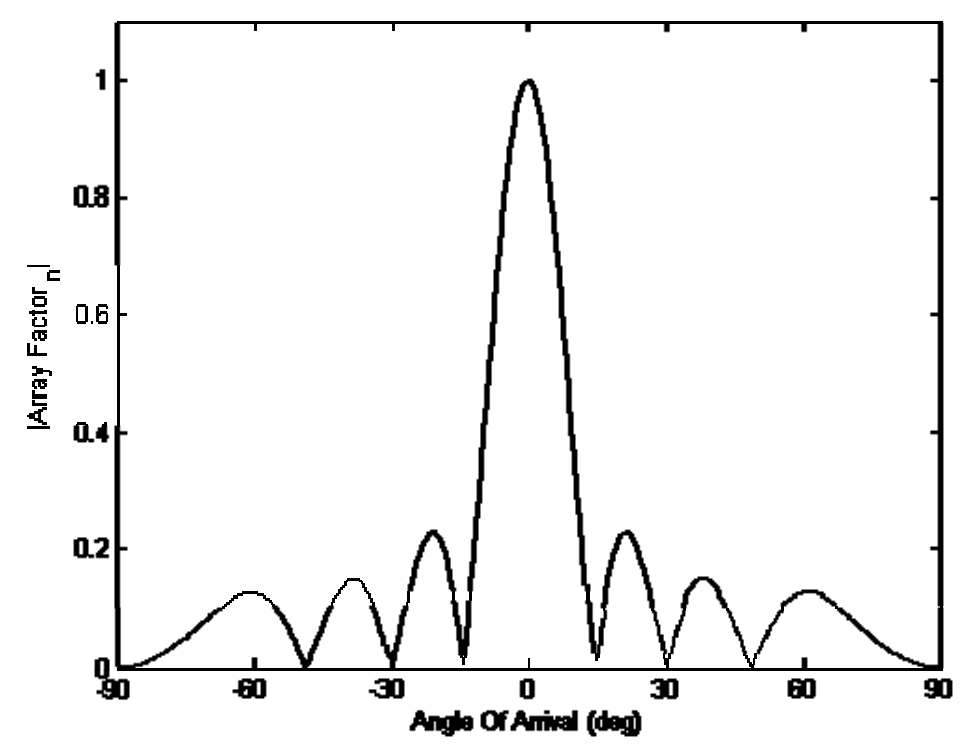

Fig.1.The array factor using LMS algorithm

The LMS algorithm contains three steps in each recursion:

$\checkmark$ Computation of the processed signal with the current set of weights.

$\checkmark$ Generation of the error between the processed signal and the desired signal.

$\checkmark$ Adjustment of the weights with the new error information by the gradient method.

In our work the Updating of weights in LMS algorithm in 8 Elements is given results and presented in Fig.3:

$$
\mathrm{W}_{1}=0.034+0.012 \mathrm{i}
$$

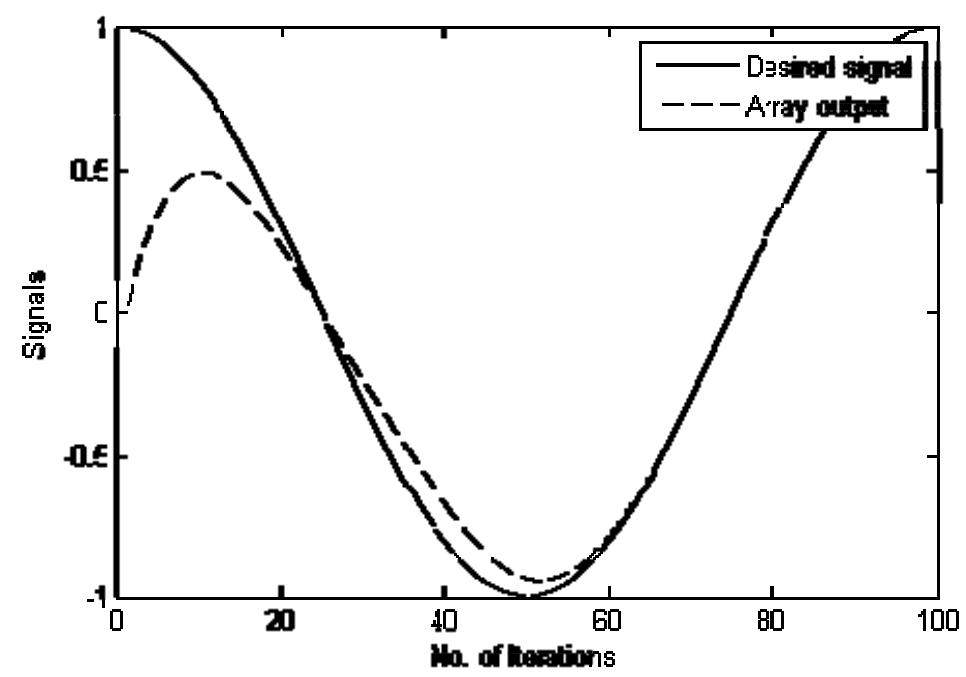

Fig.2.Acquisition and tracking of desired signal

In the Fig. 3 the result is describes the algorithmic changing the weighting in each iteration we observed that the Least Mean squares "LMS" algorithm converge after 20 iterations. We mentioned that even if the characteristic of the signal rapidly changing, the LMS algorithm may not converge after 20 iterations in a satisfactory manner. 


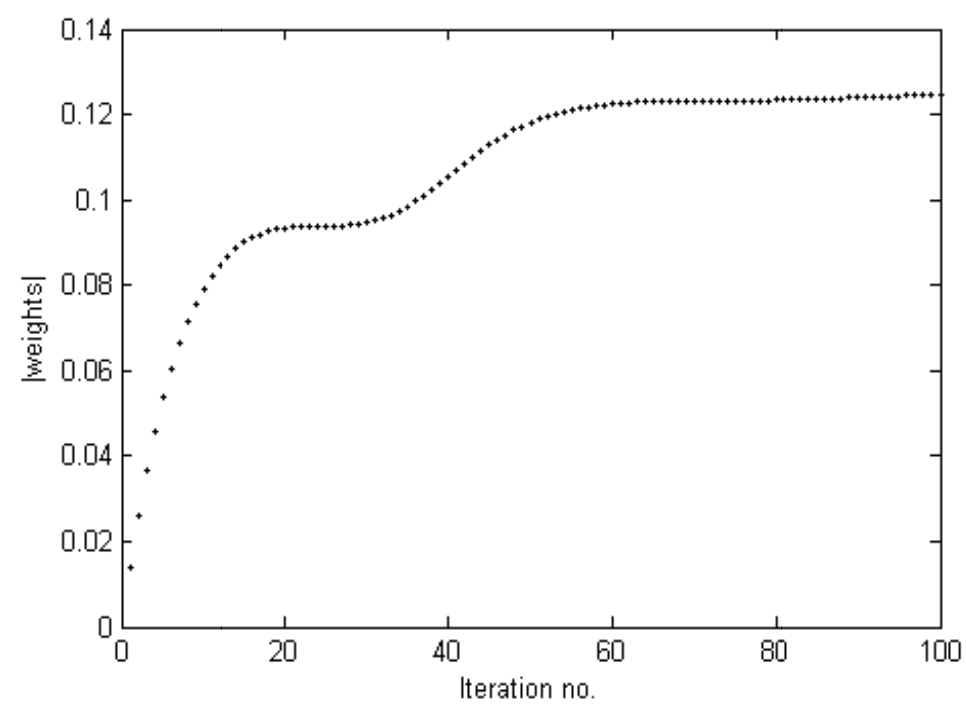

Fig.3. Magnitude of array weights using LMS algorithm

Finally, we simulated the Mean Square Error 'MSE' error in each iteration presented in Fig.4, the simulation present the relationship between mean square error and number of iteration We mentioned that even if number of iteration rapidly increases algorithm the performance of LMS MUSIC algorithm is always better than the proposed algorithm[22].

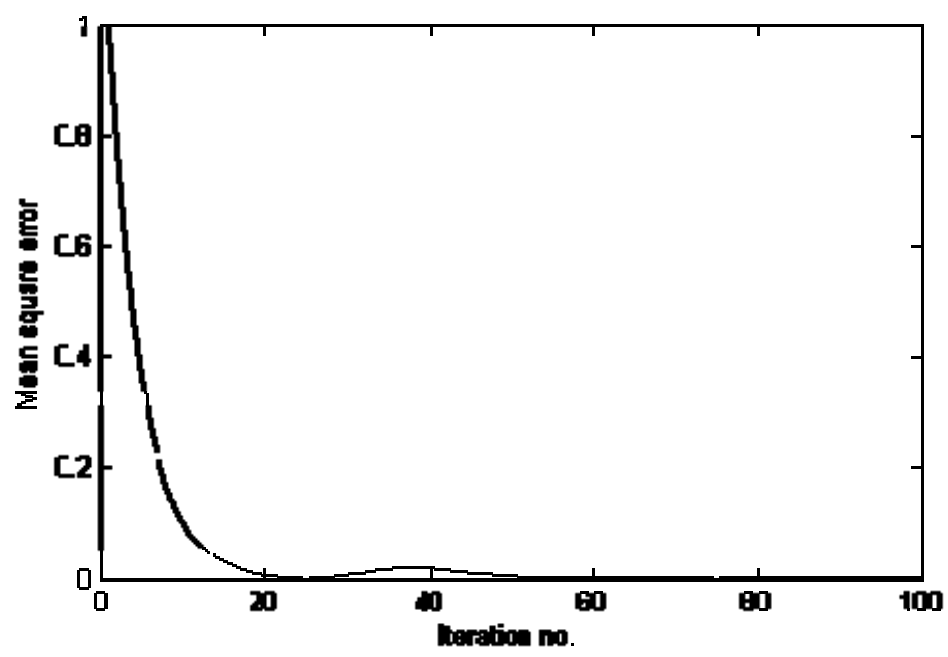

Fig.4.The mean square error using LMS algorithm

For simulation purposes the uniform circular array with $\mathrm{N}$ number of element and input signal is modulated by using BPSK modulation is considered. Simulation of LMS algorithm to illustrate how various parameters such as number of antenna element, inter element spacing parameter affect the beam formation and convergence of the algorithm.Figs.5-6 present the array factor plot of LMS algorithm when number of antenna array element is 8,10 and desired user is arriving at an angle 0 degree and interfere is at an angle of 60 degree. It has been noticed from results that sharper beams are directed towards desired signals as more elements are used in antenna array. Also, spacing between array elements has an effect on beam performance such that very small or very large spacing between array elements can degrade former performance. From different numerical calculation it has been observed that when distance between array elements is half wave length ' $0.5 \lambda$ 'then granting lobes are avoided. 


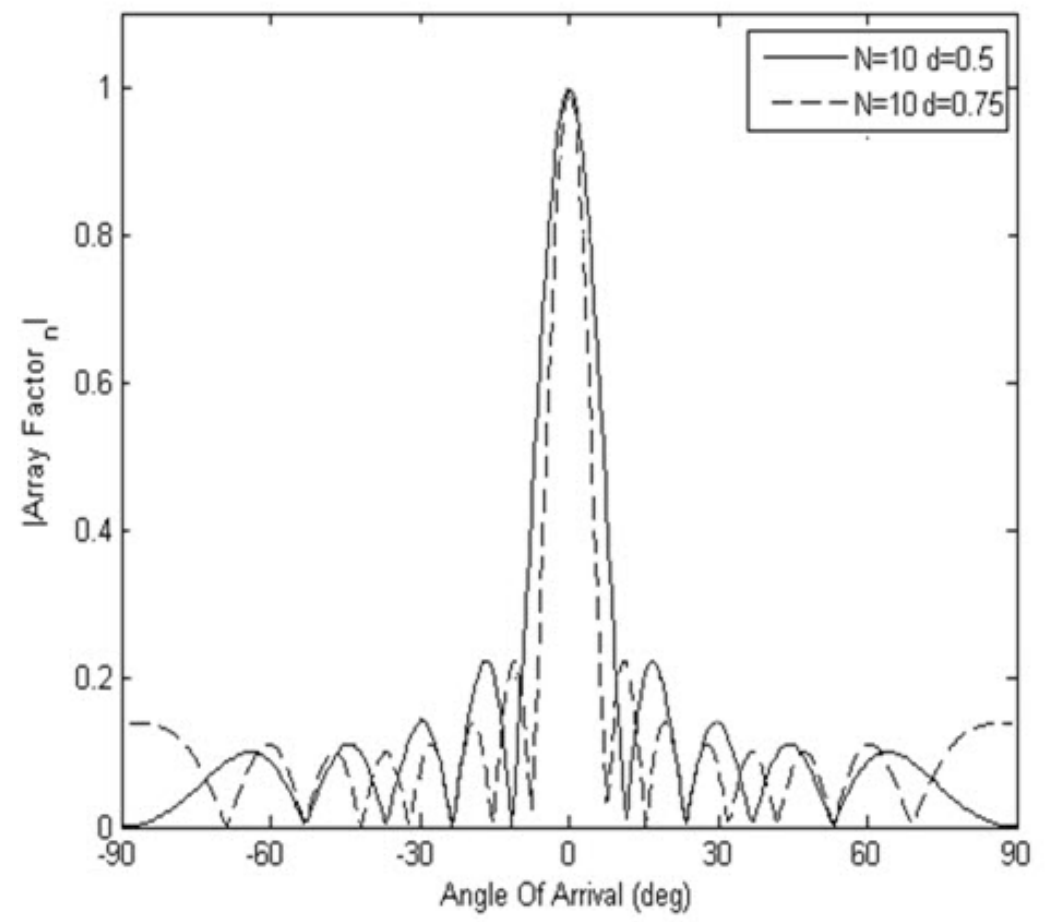

Fig.5.Array factor plot for $\mathrm{N}=10$ and 50 iteration

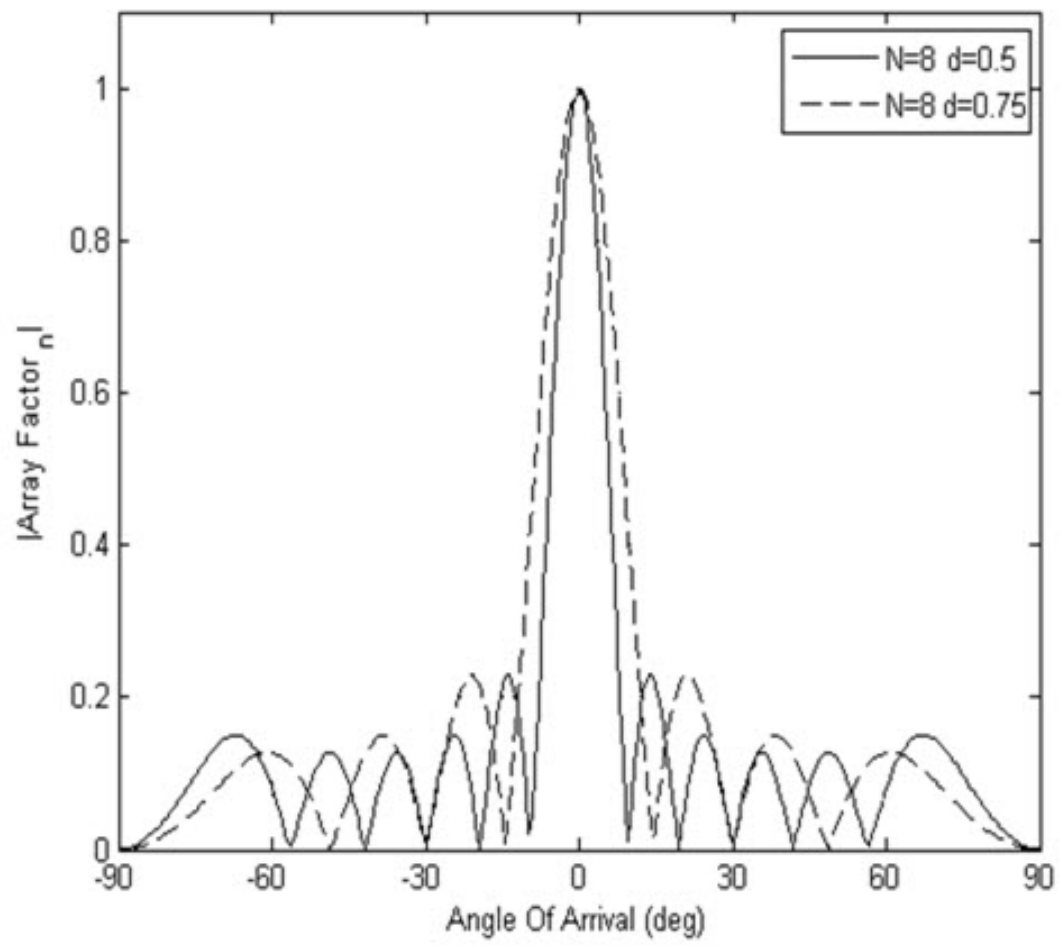

Fig.6.Array factor plot for $\mathrm{N}=8$ and 50 iteration 


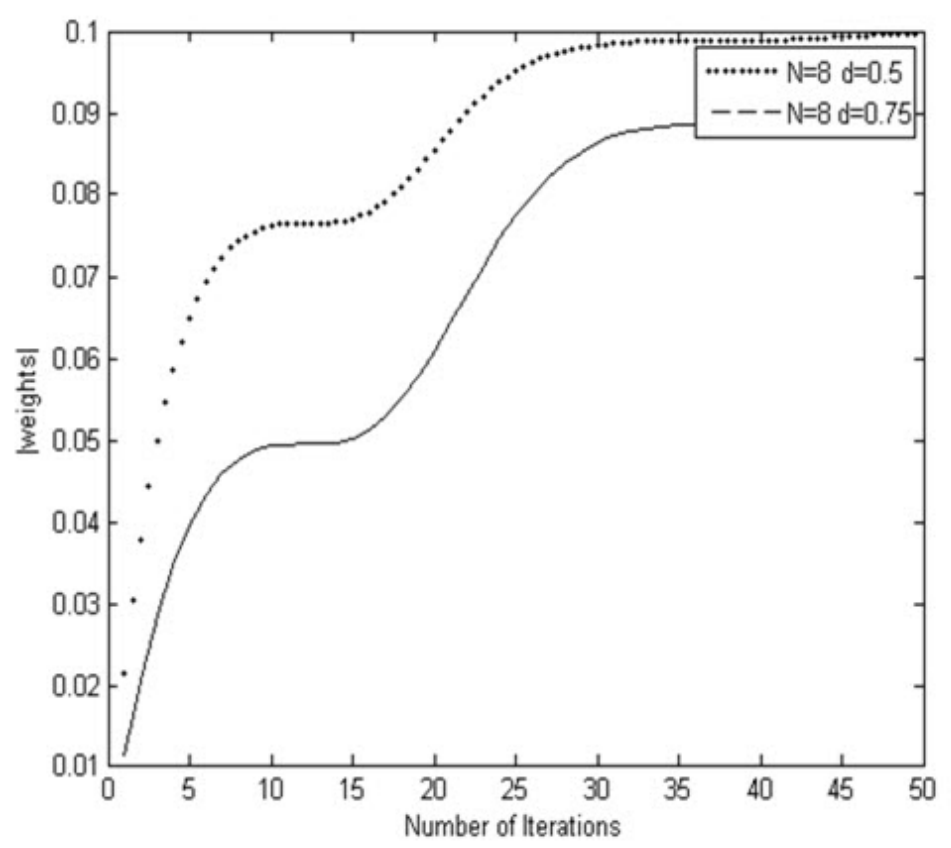

Fig.7.Weights for $\mathrm{N}=8$ and 50 iteration

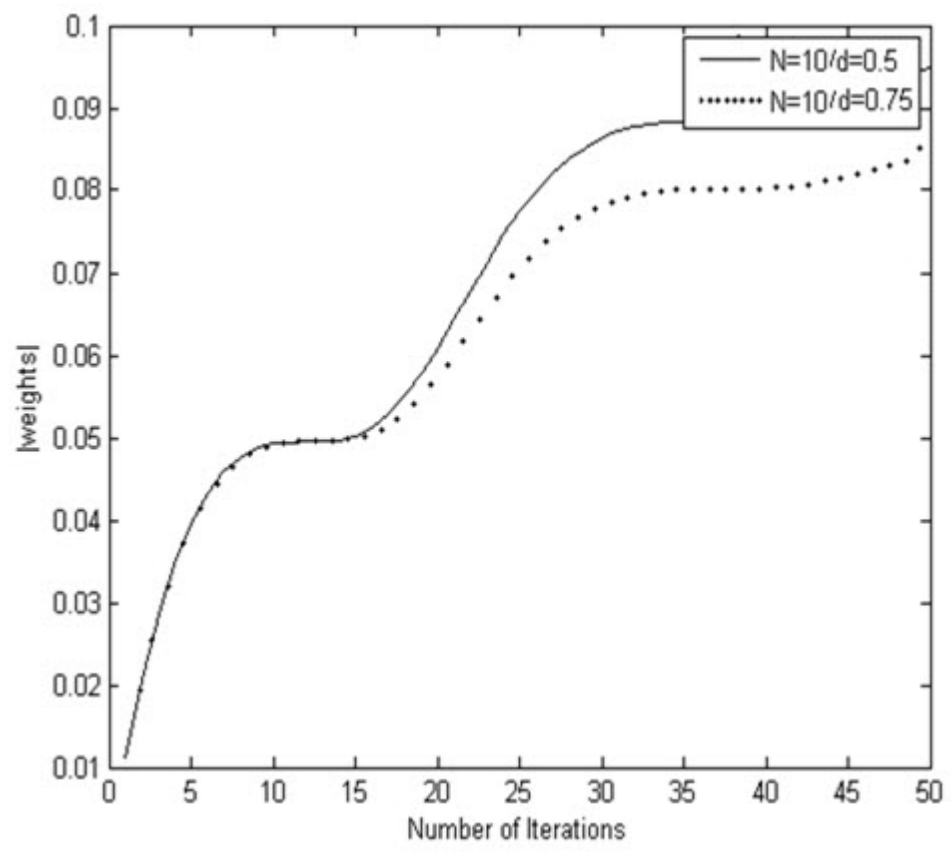

Fig.8. Weight plot $\mathrm{N}=10$ and 50 iteration

Figs.7-8 of Weight for $\mathrm{N}=8$ and spacing between array elements equal to half wavelength gives an optimum error in a particular iteration. The above graphs present The Least Mean Squares algorithm fixes the weights such that maximum directivity is achieved in given input direction when the distance between elements is equal to half wave 0.5 even if we change the number of arrays.

\section{Conclusion}

In order to improve the performance of Matrix Pencil using UCA in finite snapshots and SNR, the configurations allows us to work in real time. We analysis the performances of adaptive LMS algorithm for smart antenna systems which are very important for smart antenna design. The LMS algorithm is compared on the basis of normalized array factor and root mean square error (RMSE) for Smart Antenna systems. It is observed that an LMS algorithm is converging after 20 iterations. The attractive quality of LMS algorithm is less computational complexity then the RMSE. Our findings are explained in details above. For the UCA network, the method based on the Matrix Pencil yielded better results and the side lobe levels are considerably reduced when compared to other structure. 


\section{ACKNOWLEDGEMENTS}

This work is supported by Moulay Ismail University, Meknes - Morocco

\section{REFERENCES}

[1] LC .Godara, "Application of Antenna Arrays to Mobile Communications Part II: Beam-forming and Direction of Arrival Consideration," Proc of the IEEE, 1997, p. 1195-245.

[2] AK .Shauerman and AA .Shauerman, "Spectral-Based Algorithms of Direction-of-Arrival Estimation for Adaptive Digital Antenna Arrays," Procof the 9th international conference and seminar on Micro/Nanotechnologies and Electron Devices, 2010, Novosibirsk, Russia, p. 251-55.

[3] B Liao and SC Chan, "DOA Estimation of Coherent Signals for Uniform Linear Arrays with Mutual Coupling," Proc of the IEEE International Symposium on Circuits and Systems, 2011, Rio de Janeiro, Brazil, p. 377-80.

[4] M. Jalali, MN.Moghaddasi and AHabibzadeh. Comparing accuracy for ML, MUSIC, ROOT-MUSIC and spatially smoothed algorithms for 2 users. Procof the IEEE conference Mediterranean on Microwave Symposium (MMS), 2009, Tangiers, Morocco, p. $1-5$.

[5] H. Krim and M. Viberg, "Two decades of array signal processing research: the parametric approach," IEEE Signal. Processing. Magn, Vo. 13,pp. 67-94, 1996.

[6] Y.Hua and T.K. Sarkar, "Matrix pencil method and system poles”, Signal Processing, Vol.21, pp. 195-198, 1990.

[7] Y.Hua and T.K.Sarkar , "On SVD for estimating generalized eigenvalues of singular matrix pencil in noise," IEEE Transactions on signal processing, Vol.39, pp. 892-900,1991.

[8] A. Paulraj, R. Roy and T. Kailath, “A subspace rotation approach to signal parameter estimation”, Proc of the IEEE , 1986, p.10441045 ,

[9] N. Yilmazer, A. Seckin, and T. Sarkar, "Multiple snapshot direct data domain approach and ESPRIT method for direction of arrival estimation," Digital Signal Processing - A Review Journal, Vol. 18, pp. 561-567. 2008

[10] P. Yang, F. Yang, and Z. P. Nie, "DOA ESTIMATION WITH SUB-ARRAY DIVIDED TECH-NIQUE AND INTERPORLATED ESPRIT ALGORITHM ON A CYLINDRICAL CONFORMAL ARRAY ANTENNA,” Progress In Electromagnetics Research, PIER, pp. 201-216, 2010

[11] MA. Ihedrane, S. Bri, "Direction of Arrival Estimation using MUSIC, ESPRIT and Maximum-Likelihood Algorithms for Antenna Arrays ,"WalailakJournal of Science and Technology (WJST), Vol. 13, pp. 491-502, 2015.

[12] A.EL fadl, S.Bri, M.Habibi, "Uniform rectangular smart antenna based on Matrix Pencil," Journal of Basic and Applied Scientific Research, pp. 3322-3329, December, 2011.

[13] R. Roy and T. Kailath, "EPRIT-estimation of signal parameters via rotational invariance techniques," IEEE Transactions on Signal Processing, Vol. 37, pp. 984-995, 1989.

[14] N. Yilmazer, J. Koh, and T. K. Sarkar, "Utilization of a Unitary Transform for Efficient Computation in the Matrix Pencil Method to Find the Direction of Arrival," IEEE Transactions on Antennas and Propagation, Vol. 54, pp.171-181, 2006

[15] MK.Kesba, KEK Drissi, S. Lee, K. Kerroum, C.Faure, and C. Pasquier, "Comparison of Matrix Pencil Extracted Features in Time Domain and in Frequency Domain for Radar Target Classification,” International Journal of Antennas and Propagation, Vol.2014, pp.1-9,2014.

[16] T. K. Sarkar and O. Pereira, "Using the Matrix Pencil method to estimate the parameters of a sum of complex exponentials," IEEE Antennas and Propagation Magazine, Vol. 37, pp. 48-55.1995

[17] A.J. Van Der Veen, M.C. Vanderveen, A. Paulraj, "Joint angle and delay estimation using shift invariance techniques," IEEE Transactions on Signal Processing, Vol. 46, pp. 405-418,1998.

[18] N. Yilmazer, T. K. Sarkar, "2-D unitary matrix pencil method for efficient direction of arrival estimation," Digital Signal Processing, Vol. 16,pp.767-781,2006.

[19] A.EL fadl, S.Bri, M.Habibi, "Multipath Elimination using Matrix Pencil for Smart Antenna with Uniform Linear Array," European Journal of Scientific Research,Vol. 87, pp. 397-405, 2012.

[20] Yerriswamy T. and S.N. Jagadeesha, "Fault Tolerant MatrixPencil Method for Direction of Arrival Estimation,"Signal \& Image Processing: An International Journal, Vol. 2, pp.55-67,2011.

[21] D. M. MotiurRahaman, Md. MoswerHossain, Md. MasudRana, "Least Mean Square (LMS) for Smart Antenna,”Universal Journal of Communications and Network,Vol.1,pp.16-21, 2013.

[22] S.Singh, Er. M.Kaur, "A LMS and NLMS Algorithm Analysis for Smart Antenna,"International journal of advanced research in computer science and software engineering, Vol.5, pp. 380-384, 2015.

\section{AUTHOR PROFILE}

Mohammed Amine Ihedrane was born in Fes, Morocco on February 10, 1990. He received the master degree in 2014 in microelectronics from Sidi Mohamed Ben Abdellah University, Fes, Morocco. Currently he is pursuing his Ph.D. in physics and engineering in Moulay Ismail university of Meknes, Morocco, his work is mainly about wireless communications, multiuser detection, and smart antennas.

SeddikBri is a professor at Electrical Engineering Departments, High Scholl Technology (ESTM), Moulay Ismail University, Meknes- Morocco. His work was iterated in communications systems and applications in security. 